addition, the inflammation marker ESR was negatively correlated with TFH as well as with the differentiated $B$ cell subsets in female patients. Our observations indicates a role of the humoral immune response in AS.

Disclosure of Interests: None declared

DOI: 10.1136/annrheumdis-2021-eular.785

\section{OP0025 CD209+/CD14+ DENDRITIC CELLS ARE ENRICHED AND ACTIVATED AT THE SITE OF INFLAMMATION AND ARE MODULATED BY JAK/STAT SIGNALLING}

V. Marzaioli ${ }^{1,2}$, A. Floudas ${ }^{1,2}$, M. Canavan ${ }^{1,2}$, S. Wade ${ }^{1,2}$, K. Murray ${ }^{2}$, R. Mullan ${ }^{3}$, D. Veale ${ }^{1,2}$, U. Fearon ${ }^{1,2}$. Trinity Biomedical Sciences Institute, 1 Molecular Rheumatology, Dublin, Ireland; ${ }^{2}$ Centre for Arthritis and Rheumatic Diseases, SVUH, EULAR Centre of Excellence for Rheumatology, Dublin, Ireland;

${ }^{3}$ Adelaide and Meath Hospital, Department of Rheumatology, Dublin, Ireland

Background: Dendritic cells (DCs) are a heterogeneous population of professional antigen-presenting cells which are at the interface between innate and adaptive immunity. A specific subset of DCs is known to derive from monocyte and has a key role in inflammation and infection.

Objectives: This study aimed to characterize the phenotype and function of a distinct $\mathrm{CD} 209^{+} / \mathrm{CD} 14^{+} \mathrm{DC}$ subset in the periphery and at the site of inflammation in patients with rheumatoid (RA) and psoriatic arthritic (PsA), in addition to examining the effect Tofacitinib and TNF inhibitor on their development.

Methods: Peripheral blood and synovial fluid mononuclear cells (PBMC and SFMC) were isolated by Ficoll density gradient from healthy subject $(\mathrm{HC})$, and patients with RA and PsA. Single cell synovial tissue suspension (ST) from RA and PsA patients were also established using enzymatic/mechanical digestion. PBMC, SFMC and ST were analysed by flow cytometry to identify the CD $209^{+} /$ CD14 ${ }^{+}$DC subset, its frequency and the cell surface expression of chemokines receptors (CCR6, CCR7, CXCR3, CXCR4 and CXCR5) and activation markers (CD40 and CD80). In addition, PBMC were stimulated with different TLR (LPS, CPG, R848, Poly I:C) and intracellular staining for IL $12, \operatorname{TNF} \alpha$, IL $1 \beta$ and IL6 was performed by flow cytometry. Lineage negative cells (CD3/CD19/CD56") were stimulate with GMCSF/IL4 in the presence or absence of the JAK/STAT inhibitor Tofacitinib or the TNF inhibitor Humira, and the CD209+/CD14+ DC development was evaluated by flow cytometry.

Results: We identified, for the first time, a distinct CD209+/CD14+ DC population in PBMC of patients with RA and PsA, with similar frequency across the groups. However, when PBMC were stimulated with TLRs, an increase of IL12 and TNF $\alpha$ was observed in RA and PSA PBMC when compared to HC. Interestingly, this distinct DC population was significantly enriched at the site of inflammation, in both SFMC and ST, displaying a more mature phenotype, evident by the observed significant increase in CD40 and CD80 expression. SPICE analysis further identified differential expression and co-expression of chemokine receptors at the periphery of RA and PsA patients, when compared to the HC. Furthermore synovial tissue single cell analysis from RA/PsA demonstrated a unique chemokines receptors profile demonstrating increased single expression and co-expression of CXCR3 and CXCR5 compared to periphery. Finally, we have previously observed that JAK/STAT is involved in monocyte-derived dendritic cells population development $(1,2)$, therefore we performed CD3, CD19 and CD56 depletion of RA/PsA PBMC followed by stimulation with GMCSF/IL4, to spike the Mo-DC population, in the presence of Tofacitinib or Humira. Interestingly, we observed that JAK/STAT inhibition, but not TNF inhibitor, reduced the generation and development of CD209+/ CD14+ DC.

Conclusion: We identify for the first time a distinct monocyte-derived DC population characterized as $\mathrm{CD} 209^{+} / \mathrm{CD} 14^{+}$in the periphery of RA and PsA patients. This population was enriched at the site of inflammation and displayed a unique chemokine receptor profile and activation markers, suggesting that these cells are already activated in the periphery of IA patients, and are recruited and further activated in the inflamed joint. In addition, we showed that the CD209 ${ }^{+} / C D 14^{+}$DC development is regulated by JAK/STAT signalling, but not TNF inhibition.

REFERENCES:

[1] Marzaioli V, Canavan M, Floudas A, et al. Monocyte-Derived Dendritic Cell Differentiation in Inflammatory Arthritis Is Regulated by the JAK/STAT Axis via NADPH Oxidase Regulation. Front. Immunol. 2020;11:1406.

[2] Marzaioli V, Hurtado-Nedelec M, Pintard C, et al. NOX5 and p22phox are 2 novel regulators of human monocytic differentiation into dendritic cells. Blood. 2017;130(15):1734-1745.

Acknowledgements: The authors also wish to thank all the patients who volunteered to participate into this study and the funding

Disclosure of Interests: Viviana Marzaioli: None declared, Achilleas Floudas: None declared, Mary Canavan: None declared, Siobhan Wade: None declared, Kieran Murray: None declared, Ronan Mullan: None declared, Douglas Veale Speakers bureau: Abbvie, Janssen, Novartis, MSD, Pfizer, UCB, Consultant of: Abbvie, Janssen, Novartis, MSD, Pfizer, UCB, Grant/research support from:
Janssen, Abbvie, Pfizer, UCB, Ursula Fearon Speakers bureau: Abbvie, Grant/ research support from: Janssen, Abbvie, Pfizer, UCB

DOI: 10.1136/annrheumdis-2021-eular.2122

\section{\begin{tabular}{l|l} 
OP0026 & IGF1R DEPENDENT CELL INTERACTION AND
\end{tabular} REGULATION OF AUTOANTIBODY PRODUCTION IN RHEUMATOID ARTHRITIS}

S. Erdogan ${ }^{1}$, M. Erlandsson ${ }^{1,2}$, N. Oparina ${ }^{1}$, C. Lundquist ${ }^{1}$, C. Wasen ${ }^{1}$, M. Svensson ${ }^{1}$, M. Bemark ${ }^{3}$, K. M. Andersson ${ }^{1}$, M. I. Bokarewa ${ }^{1,2} .{ }^{1}$ Gothenburg University, Rheumatology and Inflammation Research, Göteborg, Sweden; ${ }^{2}$ Sahlgrenska University Hospital, Rheumatology Clinic, Göteborg, Sweden; ${ }^{3}$ Göteborg University, Microbiology and Immunology, Göteborg, Sweden

Background: The insulin-like growth factor 1 receptor (IGF1R) signalling mediates numerous developmental processes acting through downstream adaptor molecules IRS $1 / 2$, which activate Akt and inhibit the family of forkhead box class O (FoxO). Inhibition of IGF1R signalling alleviates rheumatoid arthritis (RA) (Erlandsson et al., 2017), however, the role of IGF1R signalling in the regulation of immune function is poorly understood.

Objectives: To investigate the link between IGF1R signalling and antigen presentation in experimental arthritis.

Methods: Arthritis was induced by immunising Balb/c mice with methylated bovine serum albumin ( $\mathrm{mBSA}, \mathrm{n}=18$ ) and $\mathrm{DBA} / 1$ mice with type II collagen (CII, $\mathrm{n}=18$ ). The mice were treated with a synthetic IGF1R inhibitor NT157 or with short hairpin RNA targeting IGF1R (shIGF1R) from the day prior to immunisation. Controls were treated with cyclodextrine vehicle/ non-targeting (nt)RNA, respectively. Flow cytometry was used for spleen cell phenotype. Antibody levels were measured by ELISA. Immunohistochemistry (IHC) of spleen was performed for assessment of marginal zone (MZ) and location of $\mathrm{pS}^{612} \mathrm{RS} 1+$ and $\mathrm{pS}^{256} \mathrm{FoxO} 1+$ cells. IHC images were acquired by fluorescent confocal microscopy, and analysed using ZEN2009 and Cell Profiler soft ware.

Results: The inhibition of IGF1R resulted in an $80 \%$ increase in MZ area in NT157 treated mice compared to controls $(\mathrm{p}=0.0001)$. This was supported by a significant increase of CD21+ $(p=0.034)$ and CD23+ cell populations $(p=0.00059)$, both among the CD19+ B cells and antigen-presenting MHCII+CD19-cells, implying that IGF1R expression regulates the populations of $\mathrm{MZ}$ and follicular cells. Additionally, there was a strong positive correlation between the decrease of IGF1R+ and ICOSL+ population on CD21+ cells $(r=0.70, p=0.0071)$, which retained them in the $\mathrm{MZ}$ and prolonged communication with macrophages. Insufficient feedback from ICOSL- B cells limited expression of CXCR5 on CD4 cells. The IHC analysis displayed that, IGF1R inhibition led to abundance of inactivate $\mathrm{pS}^{612} \mathrm{IRS} 1+$ and $\mathrm{pS}^{256} \mathrm{FoxO} 1+$ cells within the $\mathrm{MZ}$, compared with controls $(\mathrm{p}=0.0002)$. Alongside the increase of IgM+B cell population $(p=0.0022)$, we observed significant increase in number of antigen-presenting $F 4 / 80+$ cells $(p=0.043)$ and MARCO expression $(p=0.043)$ after IGF1R intervention. Finally, the NT157- treated mice displayed a significant pleiotropic increase in IgM autoantibody production, with anti-CCP IgM ( $p=0.027)$, RF-IgM ( $p=0.0085)$, anti-DNA IgM $(p=0.066)$ and in total $\operatorname{IgM}(\mathrm{p}=0.027)$ levels, which correlated positively with $\mathrm{pS}^{256} \mathrm{FoxO} 1^{+}$cells $(r=0.51$ $\mathrm{p}=0.03)$. Levels of IgG were not changed.

Conclusion: We show that IGF1R signalling is important for immune cell communication after antigen challenge. IGF1R controls ICOSL dependent trafficking of $B$ cells through the $M Z$ and facilitates interaction with $T$ cells. Retention of $B$ cells in the MZ tips the balance from $T$ cell to macrophage-dependent processes, which permits the formation of autoantibody producing B cells.

\section{REFERENCES:}

[1] Erlandsson, M., et.al., 2017. IGF-1R signalling contributes to IL-6 production and $\mathrm{T}$ cell dependent inflammation in rheumatoid arthritis. Biochimica et Biophysica Acta (BBA) - Molecular Basis of Disease, 1863(9), pp.2158-2170. Disclosure of Interests: None declared DOI: 10.1136/annrheumdis-2021-eular.2440

\section{OP0027 \\ AS-RELATED TCR BETA CLONOTYPES ARE PRESENT IN DIFFERENT INFLAMED TISSUES OF PATIENTS WITH SPONDYLOARTHROPATHIES}

E. Komech ${ }^{1}$, A. Barinova ${ }^{1}$, E. Shmidt ${ }^{2}$, T. Korotaeva ${ }^{3}$, A. Koltakova ${ }^{3}$, N. Nikitina ${ }^{4}$, E. Belousova ${ }^{4}$, M. Shapina ${ }^{5}$, S. Rodionovskaya ${ }^{6}$, I. Nikishina ${ }^{7}$, S. Vahlyarskaya ${ }^{8}$, D. Atarshchikov ${ }^{9}$, E. Klescheva ${ }^{10}$, D. Chudakov ${ }^{1,11}$, S. Lukyanov ${ }^{12}$, I. Zvyagin ${ }^{1,11}$. ${ }^{1}$ Pirogov Russian National Research Medical University, Institute of Translational Medicine, Center for Precision Genome Editing and Genetic Technologies for Biomedicine, Moscow, Russian Federation; ${ }^{2}$ Pirogov Russian National Research Medical University, City Clinical Hospital \#1, Moscow, Russian Federation; ${ }^{3}$ Nasonova Research Institute of Rheumatology, Lab of spondyloarthritis and psoriatic arthritis, Moscow, Russian Federation; ${ }^{4}$ Moscow Regional Research Clinical Institute, Gastroenterology Department, Moscow, Russian Federation; ${ }^{5}$ State Scientific Center of Coloproctology, Gastroenterology Department, Moscow, Russian Federation; ${ }^{6}$ Children's Clinical Hospital of 
the Federal Medical-Biological Agency No.38, Rheumatology Department, Moscow, Russian Federation; ${ }^{7}$ Nasonova Research Institute of Rheumatology, Pediatric, Moscow, Russian Federation; ${ }^{8}$ Pirogov Russian National Research Medical University, Russian Children's Clinical Hospital, Moscow, Russian Federation; ${ }^{9}$ Central Clinical Hospital under President Affairs, Opthalmology, Moscow, Russian Federation; ${ }^{10}$ Medical Academy of Postgraduate Education, Ophtalmology, Moscow, Russian Federation; ${ }^{11}$ Shemyakin-Ovchinnikov institute of bioorganic chemistry, Genomics of adaptive immunity, Moscow, Russian Federation; ${ }^{12}$ Pirogov Russian National Research Medical University, Head of the University, Moscow, Russian Federation

Background: Recently a group of T-cell clones with characteristic T-cell receptor (TCR) motif was identified in peripheral blood and synovial fluid of HLA-B ${ }^{\star 2} 2+$ patients with ankylosing spondylitis (AS) [1-2] - a prototypic disease from a wider group of spondyloarthropathies (SpAs). Extraarticular manifestations of AS could involve skin, intestine or eye. Emerging data indicate linkage between intestinal and joint inflammation, including expression of gut-associated integrins on synovial T-cells [3-4]. However, clonal T-cell composition and presence of identical clones in different inflamed sites in SpAs remains poorly studied.

Objectives: To investigate clonal T-cell repertoire and presence of AS-related TCR motif in different sites of inflammation of patients with SpA.

Methods: Samples of synovial fluid (SF) were obtained from HLA-B*27+ and HLA$B * 27-$ patients with ankylosing spondylitis (AS) and psoriatic arthritis (PSA), as well as gut biopsy samples from patients with AS and Crohn's disease (AS/CD) or ulcerative colitis (AS/UC), and conjunctival swabs from patients with uveitis (Uv) and with or without articular manifestations (Table 1). Also SF and gut biopsy samples were obtained from HLA-B ${ }^{\star} 27$ + patients with juvenile idiopathic arthritis (JIA). For one patient PsA patient paired samples of SF and gut biopsy were obtained.

Table 1. Detection of the AS-related motif TRBV9_CASS[V/A/L/P][G/A] [L/T/V][F/Y]STDTQYF TRBJ2-3 in bTCR repertoires of samples from different inflamed sites of patients with SpA

\begin{tabular}{lcccc}
\hline Tissue & Diagnosis & B27+ & B27- & $\begin{array}{c}\text { AS-related TCR motif+ among all } \\
\text { samples from B27+ donors }\end{array}$ \\
\hline Synovial fluid & $\begin{array}{l}\text { AS } \\
\text { PSA } \\
\text { JIA }\end{array}$ & 20 & 12 & \\
Intestinal biopsy & $\begin{array}{c}\text { AS/CD } \\
\text { AS/UC } \\
\text { CLA }\end{array}$ & 4 & 3 & $3 / 4$ \\
Conjunctival swab & \begin{tabular}{l} 
Uv \\
\hline
\end{tabular} & 8 & 0 & $4 / 8$ \\
\hline
\end{tabular}

SF and gut samples were processed to isolate mononuclear cells, while conjunctival swabs were directly lysed in the lysis buffer. CD3+ $\beta 7$-intergin+ cells were isolated from SF by fluorescence-activated cell sorting. Deep TCR repertoire profiling was carried out using UMI-based cDNA library preparation technology [1]. Results: Identical T-cell clonotypes were detected between paired SF and gut samples of the same patient with psoriatic arthritis and intestinal inflammation. The subpopulation of $\beta 7$-intergin + SF T-cells shared significantly more identical clonotypes with gut biopsy repertoire compared to the bulk SF T-cell repertoire. Clonotypes belonging to the AS-related TCR beta motif TRBV9_CASS[V/A/L/P][G/A] [L/T/[F/Y]STDTQYF TRBJ2-3 were detected in all inflamed tissues tested: synovial fluid, intestinal biopsies and conjunctival swabs of SpA patients (Table 1). Importantly, we observed these clonotypes exclusively in samples from HLA-B ${ }^{\star} 27+$ donors $(n=26)$, but not in HLA-B27- context $(n=15)$ with comparable analysis depth, thus confirming strong HLA-B ${ }^{\star 27-r e s t r i c t i o n ~ o f ~ t h e ~ c l o n o t y p e s . ~ T h e ~ A S-r e l a t e d ~ c l o n o t y p e s ~ w e r e ~ d e t e c t e d ~}$ in the subpopulation of $\beta 7$-intergin+ SF T-cells from HLA-B ${ }^{\star} 27+$ patients with PsA.

Conclusion: For the first time we directly report the T-cell clonal sharing between synovial fluid and inflamed gut tissue of SpA patients. In sum our data suggests involvement of identical T-cell clones in inflammation in different anatomical sites in SpA. REFERENCES:

[1] Komech et al. Rheumatology (Oxford). 2018;57(6):1097-1104.

[2] Faham et al. Arthritis Rheumatol. 2016;11(10):300-308.

[3] Guggino et al.Ann Rheum Dis. Published Online First: 18 October 2019. doi:10.1136/annrheumdis-2019-216456.

[4] Qaiyum et al Ann Rheum Dis. 2019;78(11):1566-1575.

Acknowledgements: We thanks all the patients and medical personnel involved in the study

Disclosure of Interests: None declared

DOI: 10.1136/annrheumdis-2021-eular.3535

\section{OP0028}

CD206+CD163+ PATHOGENIC MACROPHAGES ENRICHED IN RHEUMATOID ARTHRITIS SYNOVIAL TISSUE WITH DISTINCTTRANSCRIPTIONAL SIGNATURES

M. Hanlon ${ }^{1}$, M. Canavan ${ }^{1}$, Q. Song ${ }^{2}$, N. Neto ${ }^{3}$, P. Gallagher ${ }^{4}$, R. Mullan ${ }^{5}$, C. Hurson ${ }^{6}$, M. Monaghan ${ }^{3}$, S. Nagpal ${ }^{2}$, D. Veale ${ }^{4}$, U. Fearon ${ }^{1}{ }^{1}$ Trinity
Biomedical Sciences Institute, Molecular Rheumatology Research Group, Dublin, Ireland; ' Janssen Research and Development Spring House, Immunology \& Discovery Sciences, Spring House, United States of America; ${ }^{3}$ Trinity Biomedical Sciences Institute, Mechanical and Manufacturing Engineering, Dublin, Ireland; ${ }^{4}$ St. Vincent's University Hospital, Centre for Arthritis \& Rheumatic Diseases, Dublin, Ireland; ${ }^{5}$ Tallaght Hospital, Department of Rheumatology, Dublin, Ireland; ${ }^{6}$ St. Vincent's University Hospital, Department of Orthopaedics, Dublin, Ireland

Background: Synovial tissue macrophages are an exquisitely plastic pool of innate cells that play a key role in RA disease progression. However, the precise nature, diversity, and function of macrophage subsets within the inflamed joint remains unexplored.

Objectives: Therefore, the aims of this study are to phenotypically, transcriptionally and functionally characterise synovial tissue macrophages residing within the inflamed joint.

Methods: Rheumatoid Arthritis, Psoriatic Arthritis, Osteoarthritis and healthy control synovial-tissue biopsies and synovial-fluid mononuclear cells were analysed using the following panel (CD40,-CD45,-CD64,-CD68,-CD163,-CD206,-CD253,CCR4,-CCR7,-CXCR1,-CXCR3). CD206+CD163+ and CD206-CD163- macrophages were sorted from RA synovial-tissue by FACSAria sorter; RNAseq and FLIM analysis, autologous T-cell co-culture and heathy fibroblast experiments performed. Cytokine expression was measured by MSD immunoassay.

Results: RA synovial tissue and fluid macrophages display markers typical of both M1 (CD40+CD253+) and M2 (CD206+CD163+) macrophages with a spectrum of macrophage activation states identified. Within this spectrum, significant enrichment of dominant CD206+CD163+ macrophage-subtype is present in synovial tissue versus fluid $(p<0.05)$. CD206+CD163+ synovial tissue macrophages express significantly more CD40 than synovial fluid $(p<0.0003)$, positively correlate with disease activity $(r=0.6, p<0.01)$, with baseline levels predicting response to therapy $(\mathrm{p}<0.05)$. Moreover, CD206+CD163+CD40+ macrophages are enriched in RA synovial tissue compared to PsA and OA pathotypes $(p<0.05)$. While the CD206+CD163+ subset is present in healthy synovial tissue, expression of CD40 is completely absent in healthy synovium $(p<0.05)$ with dramatically decreased expression of CX3CR1 on RA macrophages. RNAseq analysis indicates that CD206+CD163+ population is transcriptionally distinct from synovial tissue CD206-CD163-, synovial fluid CD206+CD163+, and RA monocyte-derived M1/M2 macrophages, with unique tissue-resident gene signatures. Moreover, differing metabolic demands between CD206+CD163+ and CD206-CD163- subsets was demonstrated by RNAseq and FLIM analysis. CD206+CD163+ macrophages enhance autologous T-cell responses, spontaneously secrete high levels of pro-inflammatory cytokines and activate healthy fibroblasts towards pro-inflammatory mechanisms thus further contributing to the local inflammatory response. Finally, inhibition of CD40 activity abrogates the expression of pro-inflammatory mediators (TNFa, IL-1B, IL-6, IFNy) and induces $\mathrm{IL}-10$ expression in sorted CD206+CD163+ synovial tissue-macrophages suggesting a key role for CD40 in driving this pathogenic phenotype.

Conclusion: This data identifies for the first-time enrichment of a previously undescribed dysfunctional dominant and transcriptionally distinct macrophage subtype in RA synovial tissue. Taken together, this data provides a greater understanding of the critical role tissue-resident macrophages play in perpetuating inflammation in RA. Further investigation of the molecular patterns and cues that shape specific synovial macrophage subsets may provide opportunities to reinstate RA joint homeostasis.

Disclosure of Interests: Megan Hanlon: None declared, Mary Canavan: None declared, Qingxuan Song Employee of: Janssen Research \& Development, Nuno Neto: None declared, Phil Gallagher: None declared, Ronan Mullan: None declared, Conor Hurson: None declared, Michael Monaghan: None declared Sunil Nagpal Employee of: Janssen Research \& Development, Douglas Veale Speakers bureau: Abbvie, Janssen, Novartis, MSD, Pfizer, UCB, Consultant of: Abbvie, Janssen, Novartis, MSD, Pfizer, UCB, Grant/research support from: Janssen, Abbvie, Pfizer, UCB, Ursula Fearon Speakers bureau: Abbvie, Grant/ research support from: Janssen, Abbvie, Pfizer, UCB DOI: 10.1136/annrheumdis-2021-eular.1431

\section{OP0029}

NEUTROPHIL EXTRACELLULAR TRAPS IN GIANT CELL ARTERITIS INFLAMED TEMPORAL ARTERIES: LOCALIZATION, CO-EXPRESSION OF INFLAMMATORY CYTOKINES AND ASSOCIATION WITH DISEASE EXTENSION

O. Argyropoulou ${ }^{1}$, D. Palamidas ${ }^{1}$, N. Georgantzoglou ${ }^{2}$, E. Karatza ${ }^{3}$, E. Xingi ${ }^{4}$, E. Kapsogeorgou5, C. D. Anagnostopoulos ${ }^{6}$, A. C. Lazaris ${ }^{2}$, K. Ritis ${ }^{7}$, A. Goules8, K. Kambas ${ }^{9}$, A. Tzioufas8. ${ }^{1}$ School of Medicine, National and Kapodistrian University of Athens, Pathophysiology, Athens, Greece; ${ }^{2}$ School of Medicine, National and Kapodistrian University of Athens, 1st Department of Pathology, Athens, Greece, ${ }^{3}$ National and Kapodistrian University of Athens, 2nd Propaedeutic Department of Surgery, Athens, Greece; ${ }^{4}$ Hellenic 